\title{
Semantic Image Classification for Medical Videos
}

\author{
Shih-Hsi "Alex" Liu, Yu Cao, Yili Li, Ming Li, Sanqing $\mathrm{Hu}^{*}$ \\ Department of Computer Science, California State University, Fresno, CA, USA \\ Shliu, yucao, liyili,mingli @csufresno.edu \\ *Department of Neurology, Mayo Clinic, Rochester, MN, USA \\ Hu.Sanqing@mayo.edu
}

\begin{abstract}
Due to rapid advances in video technology and biomedicine, there has been a tremendous growth in the volume of medical video data for recent years. Exploring the full potential of medical information from these data by semantic analysis is highly desirable and very useful. In this paper, we focus on how to classify images into semantic categories effectively and efficiently. There are two major contributions in this paper. The first contribution is that our proposed approach performs classification without segmentation and processing of individual objects. The second contribution is the proposed multiclass boosting algorithms that utilize the common features which can be shared among different semantic categories. Experimental results have demonstrated that our method is a promising strategy to solve the semantic classification problem for medical video data. To the best of our knowledge, no similar research has been reported in the biomedical image computing field and we expect our research could provide useful insights for further investigation.
\end{abstract}

\section{Introduction}

Imaging and video technologies are being incorporated into today's medical research and education. A tremendous number of medical images and videos are captured and recorded in digital format during the clinic practice. Important medical knowledge is presented in these data. For example, medical videos presenting diagnostic and therapeutic procedures are valuable for reviewing the causes of complications and the diagnosis of diseases. Medical images that illustrate a variety of shapes of the lung nodule can serve as effective teaching materials for medical training. In this paper, we concentrate our efforts on educational medical videos, which have been widely used in schools and hospitals for the training of medical students, residents, and fellows. The goal of our research is to build a machine vision system that could automatically determine the scene category of any image extracted from the video. In our research, we define a "scene" as an important medical procedure (e.g., a surgery procedure) or an event (e.g., a presentation of a physician, conversations between a patient and a physician). We call a scene category as a semantic category for medical video data because the semantics of these categories match with the natural expressed user intention and user perception. A more detailed description of our proposed scene categories could be found in Section 3.1.

Motivated by the successes of the content-based analysis of digital image/video [1-3], for recent years, there has been significant growth of research to solve the semantic image categorization problems for a wide range of biomedical domains. For example, Li et al. [4] propose an image segmentation method to extract the colon lumen for virtual colonoscopy. In [5], image segmentation, feature extraction, and hierarchical cluster indexing tree are combined together to provide efficient content-based image retrieval for large biomedical image database. Liu et al. [6] present a classification-driven feature selection framework for semantic image retrieval on volumetric pathological neuroimages. Zhu et al. [7] present a video database management framework and strategies for educational medical video content structure and events mining. In [8], a multi-layered semantic analysis method that utilizes object recognition, detection, and tracking techniques is proposed for microscopic image analysis. Kumar et al. [9] introduce image partition methods for analyzing and processing large digital microscopy image datasets. In our previous papers [10, 11], image segmentation-based spatial analysis techniques are employed to locate the images with anatomy of interests in a colonoscopy video. Most of these existing methods follow the guidance from cognitive science [12]. A scene is recognized as a specific configuration of the objects. Therefore, one common theme among 
these existing methods is that these methods are heavily depending on complicated image processing techniques, such as image segmentation and object recognition. While this classic approach works in many cases, there are some potential problems associated with these methods. For example, the computation cost is high since segmentation and recognition are usually computational intensive operations. Single failure of an individual component (e.g., inaccuracy of the segmentation component, failure of the object recognition component) in the system may deteriorate the overview performance significantly. Another common characteristic among existing approaches is to pursue the class-specific features, which are compact representations for single image category. The more specific of these features, the more likely we can achieve a stronger classifier. However, as we may notice, different images from different semantic categories may share some visual properties, such as similar edges and shapes in some parts of the images. This observation prompts an interesting question: Can we take advantage of these shared features to facilitate the image classification task?

In this paper, we synthesize key ideas from [13-20] and propose a new semantic image classification method for medical video data. In this method, there are two important steps. Each of them corresponds to one of the two major contributions of this paper. The first step of our method is feature extraction. Instead of utilizing image segmentation and object detection techniques, we model the structure of the image in each semantic category using global image features. These features are generated from the statistic analysis of the power spectrum of the image signal. The power spectrum encodes the energy density for each spatial frequency and orientation over the entire image. Therefore, it provides a statistical summary of the spatial layout properties of the image. In the second step, we train a multi-class classifier based on boosted decision stumps using shared features. Instead of training an individual binary classifier for each semantic image class independently, the classifier for each category is trained jointly. The computation complexity, as well as the number of training samples, is reduced by identifying shared features among different image classes. Essentially, the two-steps method (feature extraction and multi-class classifier) is trying to solve the two issues we have mentioned in the previous paragraph: feature extraction without image segmentation or object recognition; and image classification with shared features. By applying our method to images extracted from educational medical videos, we have shown that the idea of using power spectrum analysis to model the image and employing the shared features for classification are very promising strategy to solve the semantic image classification problem.

The rest of this paper is organized as follows. Section 2 introduces the research work that is close related with our proposed approach. Section 3 presents our proposed approach. The experimental results of our methods are introduced in Section 4. Finally, we offer our concluding remarks in Section 5.

\section{Related Research}

Besides the seminal research in cognitive science (a scene is recognized by a specific configuration of the objects) [12], there is an alternative approach called "Progressive reconstruction scheme" [13] recently emerged from the vision research community. This schema builds the semantic information directly from low-level features. In addition, experiments in cognitive psychology [14] show evidence that a human being is able to recognize complex scene images even the objects have been severely degraded. Other experimental evidences $[21,22]$ also suggest that people extract enough visual information to accurately recognize its categorical properties whereas they overlook most of the features concerning the objects and their relations. These exciting discoveries have been utilized to solve the natural scene categorization problem [15, 16, 19] and promising results have been reported. Our proposed feature extraction algorithms are benefited from these discoveries.

Recently, the trend of pursuing the class-specific features to build a stronger classifier is challenged by the advances made in the areas of machine learning and computer vision [17, 18, 20]. In this work, the common features shared among different classes are employed to build a stronger classifier. Several classification frameworks, such as feedforward neural networks and boosted decision stumps, have been used in these papers. These papers motivate us to investigate new learning framework that could utilize both shared features and class-specific features to generate a stronger classifier for biomedical applications.

\section{Proposed Approach}

As we have discussed in the previous sections, our method does not rely on the computational intensive operations, such as image segmentation and object detection. Instead, we propose a semantic scene categorization method based on power spectrum analysis. In addition to that, we introduce a boosting learning framework that utilizes both shared features and class-specific features for classification. In the following sub-sections, we first introduce our research on image modeling (Section 3.1). Our modeling techniques focus on how to model the content 
difference among different semantic categories. In Section 3.2, we introduce our proposed multi-class boosting learning framework. Next, we introduce the implementation details in Section 3.3.

\subsection{Image Modeling}

Similar to other semantic categorization algorithms, the first and necessary step of our solution is to define semantic categories (i.e., scenes) in the medical video context. By consulting with the domain experts, we propose five semantic scene categories as follows: "General Introduction", "Presentation", "Conversation", "Surgery", and "Medical Procedure." The majority of the images in "General Introduction" category are natural images such as hospital buildings in the urban scene. During this scene, there is a third party anchor (not visible from the screen) that presents the highlights of the video. An example image in this category is shown in the image (a) of Figure 1. In the "Presentation" scene, the video may show the presentations given by physicians. These physicians introduce an overview of a medical procedure, as well as the explanations of some technique concepts related with the procedure. The images in this scene are usually individual physician's image captured from different view angles. This kind of image is illustrated by image (b) of Figure 1. The images that show the conversation between a physician and a patient belong to the "Conversation" scene. Image (c) of Figure 1 illustrates this scene. The image (d) of Figure 1 shows an example image for "Surgery" scene. Usually, the images in this scene include multiple objects (e.g., operation table and instrument) and people (e.g., surgeons, nurses, and patients). Besides the aforementioned images, many images in medical videos present the medical procedure whose major contents are the human anatomy (e.g., the inside of the organ and the structures in the body) and medical device (e.g., endoscopes and pacemaker). We define these images as "Medical Procedure" scene.

The next step is to investigate the power spectrum of the images from each semantic category. Our exploration is motivated by seminal studies in image science and cognitive science [23, 24] that the amplitude spectrum of natural images falls more or less monotonically with spatial frequency. Some other studies [25] also indicate that the shape of the spectra from natural images follows particular regularities. Can we find similar patterns in our biomedical images? If so, what do these patterns look like? To answer these questions, we start our investigation from power spectrum analysis for each image category. Figure 1 illustrates a part of our discoveries. The second row of Figure 1 (image (f) to image (g)) shows the

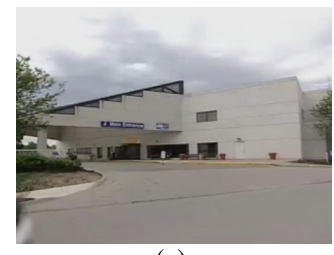

(a)

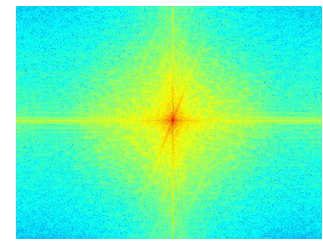

(f)

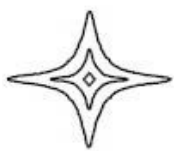

(k)

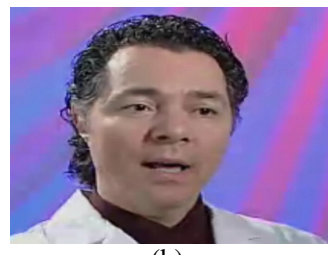

(b)

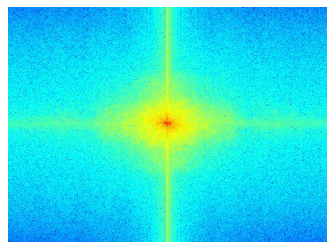

(g)

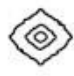

(1)

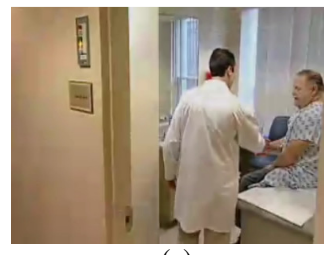

(c)

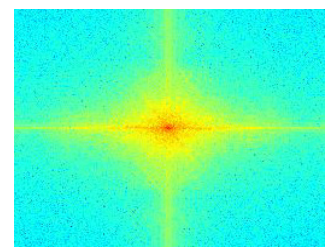

(h)

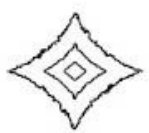

(m)

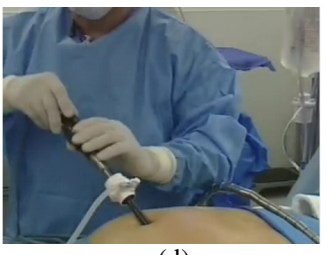

(d)

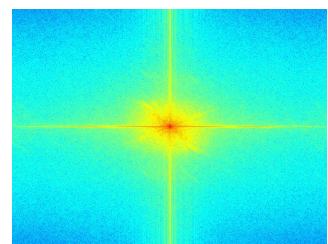

(i)

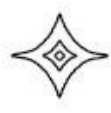

(n)

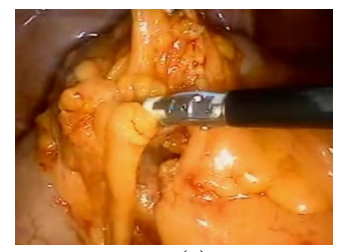

(e)

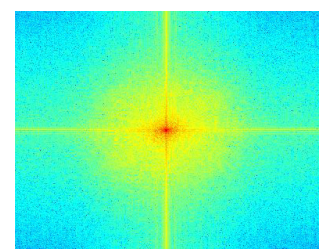

(j)

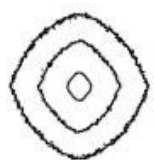

(o)

Figure 1: Image examples for each semantic category, their power spectrum, and the corresponding spectral signatures. Images in the first row are the image examples for each category: (a) "General Introduction" scene; (b) "Presentation" scene; (c)"Conversation" scene; (d) "Surgery"; and (e)"Medical Procedure" scene. Images in the second row are power spectrum images while the images in the third row are spectrum signatures. Please refer to the second paragraph of Section 3.1 for detailed description (Images are best viewed in color). 
corresponding power spectrum image of the image examples in first row of Figure 1. For example, image (f) is the power spectrum of image (a), image $(\mathrm{g})$ is the power spectrum of image (b), and so on and so forth. To further explore the hidden patterns among these images, we compute the spectral signature for each category by averaging the power spectrum from a sequence of images per category. The results are shown in the third row of Figure 1. From these image examples, we can tell that different semantic categories exhibit different orientations and spatial frequency distributions. These differences can be captured by power spectrum analysis. Specifically, most of the images in the "General Introduction" scene are urban images with buildings (e.g., hospital buildings) captured from a far distance. Consequently, the energy of the power spectrum is concentrated mainly in high spatial frequencies, which is shown in image $(\mathrm{k})$ of Figure 1. While the distance between the camera and the scene decreases, the visual field is limited to a smaller space and it is likely to produce images with flat and smooth surfaces, due to the close-up view of the objects. This visual characteristic is consistent with the visual properties of the images in the "Surgery" scene since most of the images in this scene are objects (e.g., operation table and instrument) and people (e.g., surgeons, nurses, and patients) with close-up view. In addition, the close-up view of the objects has a tendency of being isotropic in orientations. The spectral signature of "Surgery" scene, which is the image (n) of Figure 1, verifies this discovery. On the other hand, the images belong to "Medical Procedure" scene are the close-up view of the human anatomy, which shows many small grains. Therefore, the level of clutter is large and the energy is more concentrated in high frequency. Image (o) of Figure 1, which is the spectral signature for "Medical Procedure" scene, illustrates this property. The majority of images in "Presentation" scene are images with close-up view of physicians while the images in "Conversation" scene refer to the images that shooting the conversations. Usually, the "Conversation" scene refers to the images with near view of people instead of close-up view of people. This explains the spectral signature difference between image (1) and image (m) in Figure 1, which represents "Presentation" scene and "Conversation" scene, respectively.

\subsection{Multi-class boosting learning framework}

In this section, we introduce the proposed image classification algorithms based on boosting learning framework. The experts' opinions, as well as our observations, have indicated that although the content variety of the images in our research is large, there are many shared visual properties (e.g., edge like features) among different classes. In many cases, these shared features may provide similar discriminative power as the class-specific features with less training examples and training cost. The key issue is how to identify these shared features and how to incorporate these features into the classification framework. Among the state of the art classification algorithms, boosting algorithm provides a good fit for our research. Boosting is a supervised machine learning method that produces an accurate predication rule by combining rough and moderately inaccurate rules of thumb [26]. Most boosting algorithms include iteratively learning weak classifiers with respect to a distribution and add them to a final strong classifier. This iteration provides a natural interface to utilize the shared features: at each round of the boosting method, we could iterate all possible class combinations and select the combination with the largest reduction of the classification error.

Similar to existing boosting methods, our goal is to generate a strong learner $F(x, j)$ by summing up a sequence of weak learners $f_{m}(x, j)$. This could be achieved by sequentially fitting additive models of the form $F(x, j)=\sum_{m=1}^{M} f_{m}(x, j)$ where $x$ is the feature vector of the training sample, $j$ represents a semantic image class and $j \in[1, J]$ where $J$ is the number of image classes, $m$ represents the $m^{\text {th }}$ round of the boosting iteration and $M$ is the total number of boosting rounds. At each boosting round $m$, existing methods iterate $j$ times where $j$ is total number of classes. This approach does not provide any support to utilize shared features among different classes. Hinted by the technique introduced by $[18,20]$, we perform iteration for $2^{J}-1$ times instead of $J$ times at each boosting round $m$. By employing this strategy, our method could choose a subset of classes $S(m)$ that can share features and reduce classification errors. This is the key insight of our method - we traverse each (image) class and their combinations instead of traveling each class only. We can use a toy example to illustrate this insight. Suppose we have three image classes $\left\{m_{1}, m_{2}, m_{3}\right\}$. In the existing boosting algorithm, there will be three iterations at each boosting round, each of them corresponding to one class $m_{i}$ where $i \in\{1,2,3\}$. In our method, we will have seven iterations $\left(2^{3}-1=7\right)$ for each boosting round, each of them corresponding to one of the seven subsets $\left\{\left\{m_{1}\right\},\left\{m_{2}\right\},\left\{m_{3}\right\},\left\{m_{1}, m_{2}\right\},\left\{m_{1}, m_{3}\right\},\left\{m_{2}, m_{3}\right\},\left\{m_{1}, m_{2}, m_{3}\right\}\right\}$. As shown in Figure 2, we use a graph to represent 
these seven subsets and this graph is called "Sharing Graph." Each of the seven subsets is corresponding to one of the seven nodes in the sharing graph.

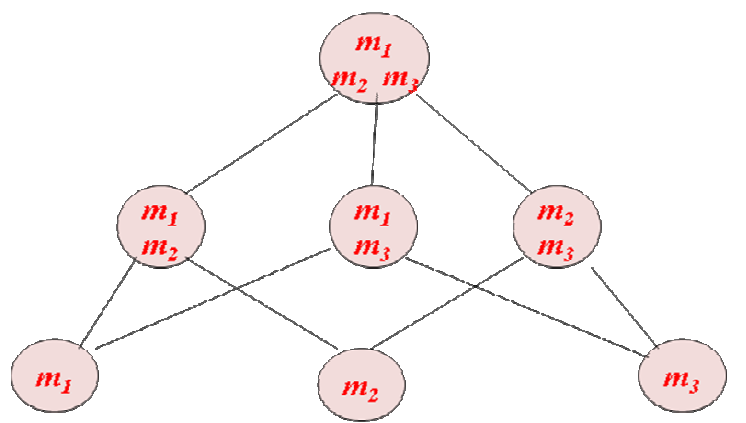

Figure 2: A graph structure (Sharing Graph) that illustrates all the possible ways to share features among three classes

Based on our framework, we will be able to search all possible subsets of individual classes and class combinations at each boosting round. This strategy guaranties the selection of best features from the feature pool that contains both shared features and class-specific features. To address the issue of searching among all the nodes in a sharing graph at each boosting round, we could use the best-search and a forward selection procedure, similar to the one introduced by $[18,27,28]$. The greedy search strategy could reduce the computation time from exponential to polynomial.

\subsection{Implementation details}

In this section, we introduce the implementation details of our proposed methods. The first step of our implementation is feature extraction. We first compute the power spectrum of an image using the discrete Fourier transform (DFT). The DFT converts an image in the spatial domain into the image in the frequency domain. Other techniques such as the maximum entropy method can also be used. In order to remove the effects of illuminance and large shadows that can hinder the detection of critical components of the image, we apply a high pass filter to the image. Then we use the Gabor filters to extract orientation and scale information from the power spectrum of the input image. The Gabor filters are chosen for this study because they allow us to make better distinctions among different categories in the frequency domain. We then use the inverse Fourier transform to recover the filtered image. Finally, we divide the output image by one quarter horizontally and vertically. There are 16 blocks for the final output. For each block, we find the mean value of pixels. Since we have 16 blocks of the image, we could find 16 feature elements for each Gabor filter. Therefore, the number of feature elements for each image will be 16 times 24 (the number of Gabor Filters we use), which is 384 . Once the features are extracted from the images, we implement the proposed boosting algorithms. One important parameter in our implementation is the number of boosting round. In our practice, we found that the value 50 gives the good balance between the training cost and classification accuracy. To compare our proposed classifier with existing classification algorithms, we implement two existing classifiers, K-nearest neighbor (K-NN) and Support Vector Machine (SVM). In our experiments, we apply these classifiers to the same data sets and we will report our results in next section.

\section{Experimental results}

Table I

Confusion Matrix of the Proposed Approach

\begin{tabular}{|c|c|c|c|c|c|}
\hline $\begin{array}{c}\text { Generation } \\
\text { Introduction }\end{array}$ & $\mathbf{0 . 9 0}$ & 0.02 & 0.03 & 0.04 & 0.01 \\
\hline Presentation & 0.01 & $\mathbf{0 . 7 8}$ & 0.12 & 0.07 & 0.04 \\
\hline Conversation & 0.03 & 0.10 & $\mathbf{0 . 7 8}$ & 0.07 & 0.02 \\
\hline Surgery & 0.03 & 0.03 & 0.06 & $\mathbf{0 . 8 2}$ & 0.10 \\
\hline $\begin{array}{c}\text { Medical } \\
\text { Procedure }\end{array}$ & 0.00 & 0.03 & 0.04 & 0.05 & $\mathbf{0 . 8 6}$ \\
\hline
\end{tabular}

To show the effectiveness of our approach, we select 1200 images that are extracted from educational medical videos, 240 images per category (five categories in total). Ten fold cross-validation is employed to test the performance of the algorithms. Table 1 shows the confusion matrix for our datasets with our proposed feature extraction methods and boosting classifier. The overall performance is encouraging with average accuracy at $83 \%$. However, the confusions between "Presentation" and "Conversation" are large. The main reason is because the visual properties between "Presentation" and "Conversation" categories are similar to each other. For performance comparison, we have conducted two types of experiments. In the first type of experiments, we implemented other scene categorization algorithms, including the "Spatial Envelope" algorithm [15] and the Baysian hierarchical model approach [29]. The average accuracies of the two algorithms in our data sets are $75 \%$ and $71 \%$, respectively. One of the reasons of the lower performance of the compared algorithms may be because both of them are proposed for natural scene categorization while our research focuses on biomedical image categorization. Another type of experiment is performed between our proposed classification method and other popular classification methods, such as K-NN and SVM, the results show that our proposed classification schema uses fewer training samples and less training time to reach similar classification accuracy as other classifiers. 


\section{Conclusions}

In this paper, we have demonstrated our investigation on modeling the medical image category directly from global features based on power spectrum analysis and classifying images using shared features. Both theoretic analysis and experimental results have suggested that it is feasible to recognize the semantic category for particular biomedical images without the segmentation and object detection. Our proposed method is a promising alternative to complement the state of the art. In the near future, we plan to refine the current implementation and apply the algorithms to larger datasets. We also plan to investigate more generic image modeling techniques that can be applied to other medical imaging/video modalities. Other topics for future investigation include extending the proposed methods to localize important medical events from video sequences.

\section{References}

[1] H. Zhang, "Content-Based Video Browsing and Retrieval," in Handbook of Multimedia Computing, vol. 5, Internet and Communications Series, B. Furht, Ed.: CRC Press, 1998, pp. 255-280.

[2] A. Hanjalic, Content-Based Analysis of Digital Video. New York, NY, USA: Springer US, 2004.

[3] R. Datta, D. Joshi, J. Li, and J. Z. Wang, "Image Retrieval: Ideas, Influences, and Trends of the New Age," $A C M$ Computing Surveys, vol. 40, pp. 1-60, 2008.

[4] L. Li, D. Chen, S. Lakare, K. Kreeger, I. Bitter, A. Kaufman, M. Wax, P. Djuric, and Z. Liang, "An image segmentation approach to extract colon lumen through colonic material tagging and Hidden Markov Random Field model for virtual colonoscopy," in Proc. of SPIE 2002 Symposium on Medical Imaging, San Diego, CA, USA, 2002.

[5] S. Antani, L. R. Long, and G. R. Thoma, "Content-based image retrieval for large biomedical image archives," in Proc. of 11th World Congress on Medical Informatics (MEDINFO), San Francisco, California, USA, 2004.

[6] Y. Liu, N. Lazar, W. Rothfus, F. Dellaert, A. Moore, J. Schneider, and T. Kanade, "Semantic-based Biomedical Image Indexing and Retrieval," in Trends and Advances in ContentBased Image and Video Retrieval, Shapiro, Kriegel, and Veltkamp, Eds.: Springer Verlag, 2002.

[7] X. Zhu, W. G. Aref, J. Fan, A. C. Catlin, and A. K. Elmagarmid, "Medical Video Mining for Efficient Database Indexing, Management and Access," in Proc. of International Conference on Data Engineering (ICDE), Bangalore, India, 2003.

[8] W. A. Ahmed, M. N. Ayyaz, B. Rajwa, F. Khan, A. Ghafoor, and J. P. Robinson, "Semantic Analysis of Biological Imaging Data: Challenges and Opportunities," International Journal on Semantic Computing, vol. 1, pp. 67-85, 2007.

[9] V. S. Kumar, S. Narayanan, T. Kurc, Jun Kong, M. N. Gurcan, and J. H. Saltz, "Analysis and Semantic Querying in Large Biomedical Image Datasets," IEEE Computer, vol. 41, pp. 5259, 2008.

[10] Y. Cao, D. Liu, W. Tavanapong, J. Wong, J. Oh, and P. C. d. Groen, "Automatic Classification of Image with Appendiceal Orifice in Colonoscopy Videos," in Proc. of the IEEE International Conference of the Engineering in Medicine and Biology Society(EMBC), New York City, NY, USA, 2006.
[11] Y. Cao, D. Liu, W. Tavanapong, J. Wong, J. Oh, and P. C. d. Groen, "Computer-aided Detection of Diagnostic and Therapeutic Operations in Colonoscopy Videos," IEEE Transactions on Biomedical Engineering,, vol. 54, pp. 12681279, 2007.

[12] I. Biederman, "Recognition-by-components: A theory of human image interpretation," Psychological Review, vol. 94, pp. 115-148, 1987.

[13] B. Schiele and J. L. Crowley, "Recognition without correspondence using multidimensional receptive field histograms," International Journal of Computer Vision, vol. 36, pp. 31-50, 2000

[14] A. Oliva and P. G. Schyns, "Coarse Blobs or Fine Edges? Evidence That Information Diagnosticity Changes the Perception of Complex Visual Stimuli," Cognitive Psychology, vol. 34, pp. 72-107, 1997.

[15] A. Oliva and A. Torralba, "Modeling the shape of the scene: a holistic representation of the spatial envelope," International Journal of Computer Vision, vol. 42, pp. 145-175, 2001.

[16] A. Torralba and A. Oliva, "Statistics of natural image categories," Network: computation in neural systems, vol. 14, pp. 391-412, 2003.

[17] Y. LeCun, F. J. Huang, and L. e. Bottou, "Learning methods for generic object recognition with invariance to pose and lighting," in Proc. of IEEE Computer Society Conference on Computer Vision and Pattern Recognition (CVPR), Washington, DC, USA, 2004.

[18] A. Torralba, K. P. Murphy, and W. T. Freeman, "Sharing features: efficient boosting procedures for multiclass object detection," in Proc. of IEEE Computer Society Conference on Computer Vision and Pattern Recognition (CVPR), Washington, DC, 2004.

[19] A. Oliva and A. Torralba, "Building the Gist of a Scene: The Role of Global Image Features in Recognition," in Visual Perception (Book Series of "Progress in Brain Research"), vol. 155, S. Martinez-Conde, S. Macknik, L. Martinez, J.-M. Alonso, and P. Tse, Eds. Amsterdam, Netherlands: Elsevier, 2006, pp. 23-36.

[20] A. Torralba, K. P. Murphy, and W. T. Freeman, "Sharing visual features for multiclass and multiview object detection," IEEE Transactions on Pattern Analysis and Machine Intelligence (PAMI), vol. 29, pp. 854-869, 2007.

[21] J. K. O'Regan, R. A. Rensink, and J. J. Clark, "Changeblindness as a result of 'mudsplashes'," Nature, vol. 398, pp. 34, 1999.

[22] R. A. Rensink, "The Dynamic Representation of Scenes " Visual Cognition, vol. 7, pp. 17-42, 2000.

[23] D. J. Field, "Relations between the statistics of natural images and the response properties of cortical cells," Journal of the Optical Society of America A, vol. 4, 1987.

[24] T. DJ, T. Y, and C. T, "Amplitude spectra of natural images," Ophthalmic Physiol Opt, vol. 12, pp. 229-232, 1992.

[25] G. J. Burton and I. R. Moorhead, "Color and spatial structure in natural scenes," Applied Optics, vol. 26, pp. 157-170, 1987.

[26] R. E. Schapire, "The boosting approach to machine learning: An overview," in Nonlinear Estimation and Classification, D. D. Denison, M. H. Hansen, C. Holmes, B. Mallick, and B. Yu, Eds. New York, NY, USA: Springer, 2003.

[27] J. Kittler, "Feature Set Search Algorithms," in Pattern Recognition and Signal Processing, C. H. Chen, Ed.: The Netherlands: Sijthoff \& Noordhoff, 1978.

[28] T. M. Mitchell, "Machine Learning," in Machine Learning: The McGraw-Hill, 1997.

[29] F.-F. Li and P. Perona, "A Bayesian Hierarchical Model for Learning Natural Scene Categories," in Proc. of IEEE Computer Society Conference on Computer Vision and Pattern Recognition (CVPR), San Diego, CA, USA, 2005. 